\title{
Galactic Gradients, Postbiological Evolution and the Apparent Failure of SETI
}

\author{
Milan M. Ćirković \\ Astronomical Observatory Belgrade, \\ Volgina 7, 11160 Belgrade-74, Serbia and Montenegro \\ E-mail: mcirkovic@aob.aob.bg.ac.yu \\ Robert J. Bradbury \\ Aeiveos Corporation, Seattle, WA 98103, USA \\ E-mail: bradbury@aeiveos.com
}

\begin{abstract}
Motivated by recent developments impacting our view of Fermi's paradox (absence of extraterrestrials and their manifestations from our past light cone), we suggest a reassessment of the problem itself, as well as of strategies employed by SETI projects so far. The need for such reevaluation is fueled not only by the failure of searches thus far, but also by great advances recently made in astrophysics, astrobiology, computer science and future studies, which have remained largely ignored in SETI practice. As an example of the new approach, we consider the effects of the observed metallicity and temperature gradients in the Milky Way on the spatial distribution of hypothetical advanced extraterrestrial intelligent communities. While, obviously, properties of such communities and their sociological and technological preferences are entirely unknown, we assume that (1) they operate in agreement with the known laws of physics, and (2) that at some point they typically become motivated by a meta-principle embodying the central role of information-processing; a prototype of the latter is the recently suggested Intelligence Principle of Steven J. Dick. There are specific conclusions of practical interest to astrobiological and SETI endeavors to be drawn from coupling of these reasonable assumptions with the astrophysical and astrochemical structure of the spiral disk of our Galaxy. In particular, we suggest that the outer regions of the Galactic disk are most likely locations for advanced SETI targets, and that sophisticated intelligent communities will tend to migrate outward through the Galaxy as their capacities of information-processing increase, for both thermodynamical and astrochemical reasons. However, the outward movement is limited by the decrease in matter density in the outer Milky Way. This can also be regarded as a possible generalization of the Galactic Habitable Zone, concept currently much investigated in astrobiology.
\end{abstract}

Keywords: astrobiology, Galaxy: evolution, extraterrestrial intelligence, physics of computation, SETI 
If you do not expect the unexpected, you will not find it; for it is hard to be sought out and difficult.

Heraclitus of Ephesos, fragment B18 (cca. 500 BC)

\section{Introduction}

Fermi's paradox ${ }^{1}$ has become significantly more serious, even disturbing, of late. This is due to several independent lines of scientific and technological advances occurring during the last $\sim 10$ years:

- Discovery of more than 150 extrasolar planets, on almost weekly basis (for regular updates see http://www.obspm.fr/planets); many of them are reported to be parts of systems with stable circumstellar habitable zones (Noble, Musielak, and Cuntz 2002; Asghari et al. 2004; Beaugé et al. 2005).

- Improved understanding of the details of chemical and dynamical structure of the Milky Way and its Galactic habitable zone (GHZ; Gonzalez, Brownlee, and Ward 2001). In particular, this includes important recent calculations showing that Earth-like planets began forming more than 9 Gyr ago and their median age is $6.4 \pm 0.7 \mathrm{Gyr}$, significantly more than the Earth's age (Lineweaver 2001; Lineweaver et al. 2004).

- Confirmation of the rapid origination of life on early Earth (e.g. Mojzsis et al. 1996); this rapidity, in turn, offers a strong probabilistic support to the idea of many planets in the Milky Way inhabited by at least the simplest lifeforms (Lineweaver and Davis 2002).

- Discovery of extremophiles and the general resistance of simple lifeforms to much more severe environmental stresses than it had been thought possible previously (e.g. Cavicchioli 2002). These include representatives of all three great domains of terrestrial life (Bacteria, Archaea, and Eucarya), showing that the number and variety of cosmic habitats for life are probably much larger than conventionally imagined.

- Our improved understanding in molecular biology and biochemistry leading to heightened confidence in the theories of naturalistic origin of life (Lahav, Nir, and Elitzur 2001; Ehrenfreund et al. 2002; Bada

\footnotetext{
${ }^{1}$ It would be most appropriately to call it Tsiolkovsky-Fermi-Viewing-Hart-Tipler's paradox (for much of the history, see Brin 1983; Kuiper and Brin 1989; Webb 2002, and references therein). We shall use the locution "Fermi's paradox" for the sake of brevity, and with full respect for contributions of the other important authors.
} 
2004). The same can be said, to a lesser degree, for our understanding of the origin of intelligence and technological civilization (e.g. Chernavskii $2000)$.

- Exponential growth of the technological civilization on Earth, especially manifested through Moore's Law and other advances in information technologies (Moravec 1988; Schaller 1997; Bostrom 2000). This includes the increased confidence in speculations about the ultimate physical limits on computation (Lloyd 2000).

- Improved understanding of feasibility of interstellar travel in both classical sense (Vulpetti 1999; Andrews 2003), and in the more efficient form of sending inscribed matter packages over interstellar distances (Rose and Wright 2004).

- Theoretical grounding for various astro-engineering/macro-engineering projects (Criswell 1985; Badescu 1995; Badescu and Cathcart 2000; Korycansky, Laughlin, and Adams 2001; McInnes 2002) potentially detectable over interstellar distances. Especially important in this respect is possible combination of astro-engineering and computation projects of advanced civilizations, like those envisaged by Sandberg (1999).

Although admittedly uneven and partially conjectural, this list of advances and developments (entirely unknown at the time of Tsiolkovsky's and Fermi's original remarks, and even Viewing's, Hart's and Tipler's subsequent reissues) testifies that Fermi's paradox is not only still with us more than half a century later, but that it is more puzzling and disturbing than ever. ${ }^{2}$ In addition, we have witnessed substantial research leading to a decrease in confidence in the so-called Carter's (1983) "anthropic" argument, the other mainstay of SETI scepticism (Wilson 1994; Livio 1999; Ćirković and Dragićević 2005 , preprint). All this is accompanied by increased public interest in astrobiology and related issues (e.g. Ward and Brownlee 2000, 2002; Webb 2002; Cohen and Stewart 2003; Dick 2003). The list above shows, parenthetically, that quite widespread (especially in popular press) notion that there is nothing new or interesting happening in SETI studies is deeply wrong.

Faced with aggravated situation vis-à-vis Fermi's paradox, the solution is usually sought in either (i) some version of the "rare Earth" hypothesis (i.e., the picture which emphasizes inherent uniqueness of our planet, and

\footnotetext{
${ }^{2}$ One is tempted to add another item of a completely different sort to the list: the empirical fact that we have survived about 60 years since invention of the first true weapons of mass destruction gives us at least a vague Bayesian argument countering the ideasprevailing at the time of Fermi and his original lunch-time question - that technological civilizations tend to destroy themselves as soon as they discover nuclear power. This is not to contest that the bigger of part of the road toward safety for humankind is still in front of us.
} 
hence uniqueness of human intelligence and technological civilization in the Galactic context), or (ii) "neo-catastrophic" explanations (ranging from the classical "mandatory self-destruction" explanation, championed for instance by von Hoerner or Shklovsky, to the modern emphasis on mass extinctions in the history of life and the role of catastrophic impacts, gamma-ray bursts, and similar dramatic events). Both these broad classes of hypotheses are unsatisfactory on several counts: for instance, "rare Earth" hypotheses reject the usual Copernican assumption (Earth is a typical member of the planetary set), and neo-catastrophic explanations usually fail to pass the non-exclusivity requirement (but see Ćirković 2004a,b). None of these are clear, straightforward solutions. It is quite possible that a "patchwork solution", comprised of a combination of suggested and other solutions remains our best option for solving this deep astrobiological problem. This motivates the continuation of the search for plausible explanations of Fermi's paradox.

Hereby, we would like to propose a novel solution, based on the astrophysical properties of our Galactic environment on large scales, as well as some economic and informational aspects of the presumed advanced technological civilizations (henceforth ATCs). In doing so, we will suggest a radically new perspective on the entire SETI endeavor.

\section{Digital perspective and the postbiological universe}

In an important recent paper, the distinguished historian of science Stephen J. Dick argued that there is a tension between SETI, as conventionally understood, and prospects following exponential growth of technology as perceived in recent times on Earth (Dick 2003):

But if there is a flaw in the logic of the Fermi paradox and extraterrestrials are a natural outcome of cosmic evolution, then cultural evolution may have resulted in a postbiological universe in which machines are the predominant intelligence. This is more than mere conjecture; it is a recognition of the fact that cultural evolution - the final frontier of the Drake Equation - needs to be taken into account no less than the astronomical and biological components of cosmic evolution. [emphasis in the original]

It is easy to understand the necessity of redefining SETI studies in general and our view of Fermi's paradox in particular in this context: for example, postbiological evolution makes those behavioral and social traits like territoriality or expansion drive (to fill the available ecological niche) which are - more or less successfully _ "derived from nature" lose their relevance. Other important guidelines must be derived which will encompass the vast 
realm of possibilities stemming from the concept of postbiological evolution. In particular, we follow the Intelligence Principle of Dick (2003), stating that

In sorting priorities, I adopt what I term the central principle of cultural evolution, which I refer to as the Intelligence Principle: the maintenance, improvement and perpetuation of knowledge and intelligence is the central driving force of cultural evolution, and that to the extent intelligence can be improved, it will be improved. [emphasis in the original]

Before we explore the logical consequences of the Intelligence Principle for SETI further, let us emphasize that the study of Dick (2003) is not an isolated instance. Very similar thinking is clearly emerging in various other fields and related to a plethora of different problems. Considerations of postbiological evolution may be fruitfully related to the megatrajectory concept of Knoll and Bambach (2000), who cogently argue that astrobiology is the ultimate field for verification or rejection of our biological concepts. In relation to the old problem of progress (or its absence) in the evolution of life on Earth, Knoll and Bambach offer a middle road encompassing both contingent and convergent features of biological evolution through the idea of a megatrajectory:

We believe that six broad megatrajectories capture the essence of vectorial change in the history of life. The megatrajectories for a logical sequence dictated by the necessity for complexity level $N$ to exist before $N+1$ can evolve... In the view offered here, each megatrajectory adds new and qualitatively distinct dimensions to the way life utilizes ecospace.

The six megatrajectories outlined by the biological evolution on Earth so far are: (i) from the origin of life to the "Last Common Ancestor"; (ii) prokaryote diversification; (iii) unicellular eukaryote diversification; (iv) multicellularity; (v) invasion of the land; and (vi) appearance of intelligence and technology. Postbiological evolution may present the seventh megatrajectory, triggered by the emergence of artificial intelligence at least equivalent to the biologically-evolved one, as well as the invention of several key technologies of roughly similar level of complexity and environmental impact, like molecular nanoassembly (Phoenix and Drexler 2004) or stellar uplifting (Criswell 1985). ATCs can be regarded as instantiations of this seventh (or possible higher) megatrajectory. It is not necessary to assume that the seventh megatrajectory represents the complete or partial abandonment of the biological material substratum of previous evolution, although that is certainly one of the options. Rather, the mode of evolution is likely to change from the Darwinian one dominating previous six megatrajectories, to a sort of aggregative, 
intentional, quasi-Lamarckian mode characteristic for highly developed cultural entities. We shall repeatedly return to this important point, which in a sense obviates further rather superficial speculation about the detailed structure of ATCs.

A natural extension of the Intelligence Principle is what can be called the digital perspective on astrobiology: after a particular threshold astrobiological complexity is reached, the relevant relations between existent entities are characterized by requirements of computation and information processing. It is related to the emergent computational concepts not only in biology, but in fundamental physics, cosmology, social sciences, etc. One particular consequence of the digital perspective, dealing with the thermodynamics of computation, we shall now argue, will allow us a glimpse of a novel view of the generic evolution of the intelligent communities in the Galactic context, including a new solution of the old Fermi's puzzle. The digital perspective also indicates that we should either entirely abandon or significantly modify Kardashev's (1964) classification of extraterrestrial intelligent communities, one of the mainstays of classical SETI studies.

What limits prospects of postbiological evolution guided by the Intelligence Principle? In order to answer this question, we need to consider limitations imposed by physics on the classical theory of computation. As almost anybody having practical experience with computers will have experienced, heat is an enemy of computation. In contrast to other obstacles and difficulties facing highly imperfect computers of today (like limited storage space, dust gathering on chips, or inefficiency of their human operators), the problem of heat dissipation is a consequence of the laws of physics. Therefore, we conjecture that this problem will remain the enemy of efficient computation for advanced technical civilizations, and that it will have a dominant effect on policy-making of such advanced societies.

Thermodynamics of computation has, historically, been motivated by Maxwell's demon "paradox" which led to great breakthroughs of Szilard, Brillouin, and Landauer. One of its most important results, often called Brillouin inequality is the fundamental property of the information content available for processing in any sort of physical system (Landauer 1961; Brillouin 1962):

$$
I \leq I_{\max },
$$

where the limiting amount of information $I_{\max }$ (in bits) processed using energy $\Delta E$ (in ergs) on the processor temperature $T$ (in $\mathrm{K}$ ) is given as

$$
I_{\max }=\frac{\Delta E}{k_{B} T \ln 2}=1.05 \times 10^{16} \frac{\Delta E}{T} .
$$

Here, $k_{B}$ is the Boltzmann constant. Obviously, computation becomes more efficient as the temperature of the heat reservoir in contact with the computer is lower. In the ideal case, no energy should be expended on cooling 
the computer itself, since that expense should be added to the energy cost of logical steps minimized by (2). The most efficient heat reservoir is the universe itself, which far from local energy sources like stars and galaxies, has the temperature of the cosmic microwave background (henceforth CMB; Wright et al. 1994)

$$
T_{\mathrm{CMB}}=2.736 \pm 0.017 \mathrm{~K} \text {. }
$$

However, this is an ideal case, since ATCs cannot have their computers in thermal equilibrium with $\mathrm{CMB}$ for astrophysical reasons. In the rest of this paper we shall investigate how close approach to this ideal case is feasible.

It has already been repeatedly suggested that our descendants, in particular if they cease to be organic-based, may prefer low-temperature, volatilerich outer reaches of the Solar System. Thus, they could create what could be dubbed "circumstellar technological zone" as different and complementary to the famous (and controversial) "circumstellar habitable zone" in which life is, according to most contemporary astrobiological views, bound to emerge. We propose to generalize this concept to the Galaxy (and other spiral galaxies) in complete analogy to GHZ (Gonzalez et al. 2001; Lineweaver et al. 2004). It is not necessary, or indeed desirable, for our further considerations to make the notion of ATCs more precise. The diversity of postbiological evolution is likely to at least match, and probably dwarf, the diversity of its biological precedent. It is one particular feature - information processing we assume common for the "mainstream" ATCs. Whether real ATCs can most adequately be described as "being computers" or "having computers" is not of key importance for our analysis; we just suppose that in either case the desire for optimization of computations will be one of important, if not the most important, desires of such advanced entities. It is already clear, from the obviously short and limited human astronautical experience, that postbiological evolution offers significant advantages in this field (Parkinson 2005).

\section{Galactic temperature gradient}

The famous article by Freeman Dyson (1960) proposing search for large-scale engineering projects (like eponymous Dyson shells) as the signposts of the presence of advanced extraterrestrial intelligence provoked much discussion henceforth. One very important contribution was the early suggestion of the distinguished computer scientist and AI pioneer Marvin Minsky (1973) in a debate following Dyson's talk at the celebrated Byurakan conference in 1971, that advanced computers would utilize the temperature of cosmic microwave background as a heat sink (3). ${ }^{3}$ This particular idea is wrong

\footnotetext{
${ }^{3}$ Parenthetically, in the same debate Minsky presciently suggested infeasibility of conventional SETI due to the impossibility of distinguishing the signal from the Gaussian
} 
in the specifics, at least for the younger and most accessible ATCs, but it gives us an important hint as to what should we be searching for. Subsequently, other astro-engineering projects - sometimes called megaprojects or macroprojects - aimed at optimization of resources at ATCs' disposal have been proposed, notably Jupiter Brains (Sandberg 1999; for early history see Bradbury 1997; Perry E. Metzger, private communication to RJB May 20, 1998) and Matrioshka Brains (Bradbury 2001).

What is the temperature of a solid body (like a Dyson shell, a Matrioshka brain, or a Jupiter brain ${ }^{4}$ in thermal equilibrium with the surrounding interstellar space? The dominant factor is the spatial distribution of the interstellar radiation field (henceforth ISRF), especially at short wavelengths (optical and UV). It can be shown that the most important way of energy transfer to a solid body in by far the largest fraction of the Galactic interstellar medium (ISM), is the absorption of photons while collisions with atoms and ions are unimportant. For example, ambiental ultraviolet flux close to the Solar circle of about $10^{10}$ photons $\mathrm{m}^{-2} \mathrm{~s}^{-1} \mathrm{~nm}^{-1}$ will deposit about a $10^{-20} \mathrm{~J} \mathrm{~s}^{-1}$ to a dust grain (with unity absorption factor, for simplicity), while collisions deposit $\simeq 10^{-26} n_{\mathrm{ISM}} \mathrm{J} \mathrm{s}^{-1}$, where $n_{\mathrm{ISM}}$ is the ISM number density in $\mathrm{cm}^{-3}$. Since on the average $\left\langle n_{\mathrm{ISM}}\right\rangle=1 \mathrm{~cm}^{-3}$, we perceive how unimportant collisions which form our laboratory definition of the "thermal equilibrium" are in the interstellar space.

Neglecting collisions, the temperature will be given by solving the radiative equilibrium equation (e.g. Dyson and Williams 1980)

$$
\int F(\lambda) Q_{\mathrm{abs}}(a, \lambda) d \lambda=\int Q_{\mathrm{abs}}(a, \lambda) B(\lambda, T) d \lambda,
$$

where $F(\lambda)$ is the energy flux of ISRF, $Q_{\text {abs }}$ is the absorption coefficient, and the Planck black-body function is given as

$$
B(\lambda, T)=\frac{2 h c}{\lambda^{3}} \frac{n_{\lambda}^{2}}{\exp \left(\frac{h c}{k T \lambda}\right)-1} .
$$

ISRF is created mainly by massive stars of Population I, concentrated in the Galactic disk. Typical value of the energy density of ISRF in vicinity of the Sun is $U=7 \times 10^{-13} \mathrm{ergs} \mathrm{cm}^{-3}$, which does not include the CMB contribution, which has $U_{\mathrm{CMB}}=4 \times 10^{-13} \mathrm{ergs} \mathrm{cm}^{-3}$. We use the conventional assumption of the exponential disk (e.g. Binney and Merrifield 1998) with the luminosity density approximated by

$$
j(R, z)=\frac{I_{0}}{2 z_{0}} \exp \left(-\frac{R}{R_{d}}-\frac{|z|}{z_{0}}\right),
$$

noise (cf. Lachmann, Newman, and Moore 2004).

${ }^{4}$ For the purpose of the present discussion, we use the placeholder "solid body" for any macroscopic body not made of gas or liquid. Thus, sizes of solid bodies we consider range roughly from $10^{-5} \mathrm{~cm}$ (an interstellar dust grain) to $10^{13} \mathrm{~cm}$ (a Dyson shell). 
where $I_{0}$ is the central surface brightness, $z_{0}$ is the scale-height, and $R_{d} \approx 3$ kpc is the disk scalelength. From this, we obtain the disk surface brightness as

$$
I(R)=\int_{-\infty}^{+\infty} j(R, z) d z=I_{0} \exp \left(-\frac{R}{R_{d}}\right),
$$

which agrees with observations in other disk galaxies. It seems clear that ISRF will decline with galactocentric distance, and thus the equilibrium temperature will decline too, enabling more and more efficient computation, as per (2). No detailed studies of the radiation field temperature distribution in the Milky Way disk exist so far, we suggest the following rough estimates.

Part of the answer can be gauged by comparison with the existent natural solid bodies in such thermal equilibrium, namely interstellar dust grains. In Figure 1, we see results of the detailed models of the Galactic temperature distribution of dust grains (Mathis, Mezger, and Panagia 1983; Cox, Krügel, and Mezger 1986). Using the same values of ISRF and correcting for the emission efficiency of larger solid objects we get the results presented in Figure 2, for computation efficiency defined as the maximal number of bytes processed per erg of the invested energy in terabytes $\left(10^{12}\right.$ bytes $)$ per erg.

In reality, we need to take into account the inhomogeneities in the interstellar medium, especially giant molecular clouds. The interiors of molecular clouds are impenetrable to short wavelength radiation, and present some of the coldest locales in the Milky Way $(T \sim 10 \mathrm{~K})$. However, the interiors of giant molecular clouds are also sites of vigorous massive star formation, so these low-temperature locales are quite irregular and transient phenomena, assembling and dissembling on timescales of $\sim 10^{6} \mathrm{yrs}$, which is probably unacceptable from the point of view of most ATCs (which we suppose stable at longer timescales by definition).

There have not been any studies of the ISRF for distances larger than about $14 \mathrm{kpc}$ (Prof. John S. Mathis, private communication). At some point for large galactocentric distances (larger than the Holmberg radius $\sim 20 \mathrm{kpc}$ ), practically all sources of ISRF are located at smaller $R$, so we can use the simplest approximation of galaxy as a point source. If with $T_{D}(R)$ we denote the temperature of a large solid object (a Dyson shell, say) at galactocentric distance $R$, a simple scaling relationship holds:

$$
\sigma T_{D}^{4}(R) \propto L_{*} R^{-2}
$$

where $\sigma$ is Steffan-Boltzmann constant, $L_{*} \approx 4.9 \times 10^{10} L_{\odot}$ the Galactic luminosity in the absorbing bands. Taking into account both (2) and (8), we obtain the general scaling relation in the outermost regions:

$$
\left(\frac{I}{E}\right)_{\max } \propto \sqrt{R}
$$




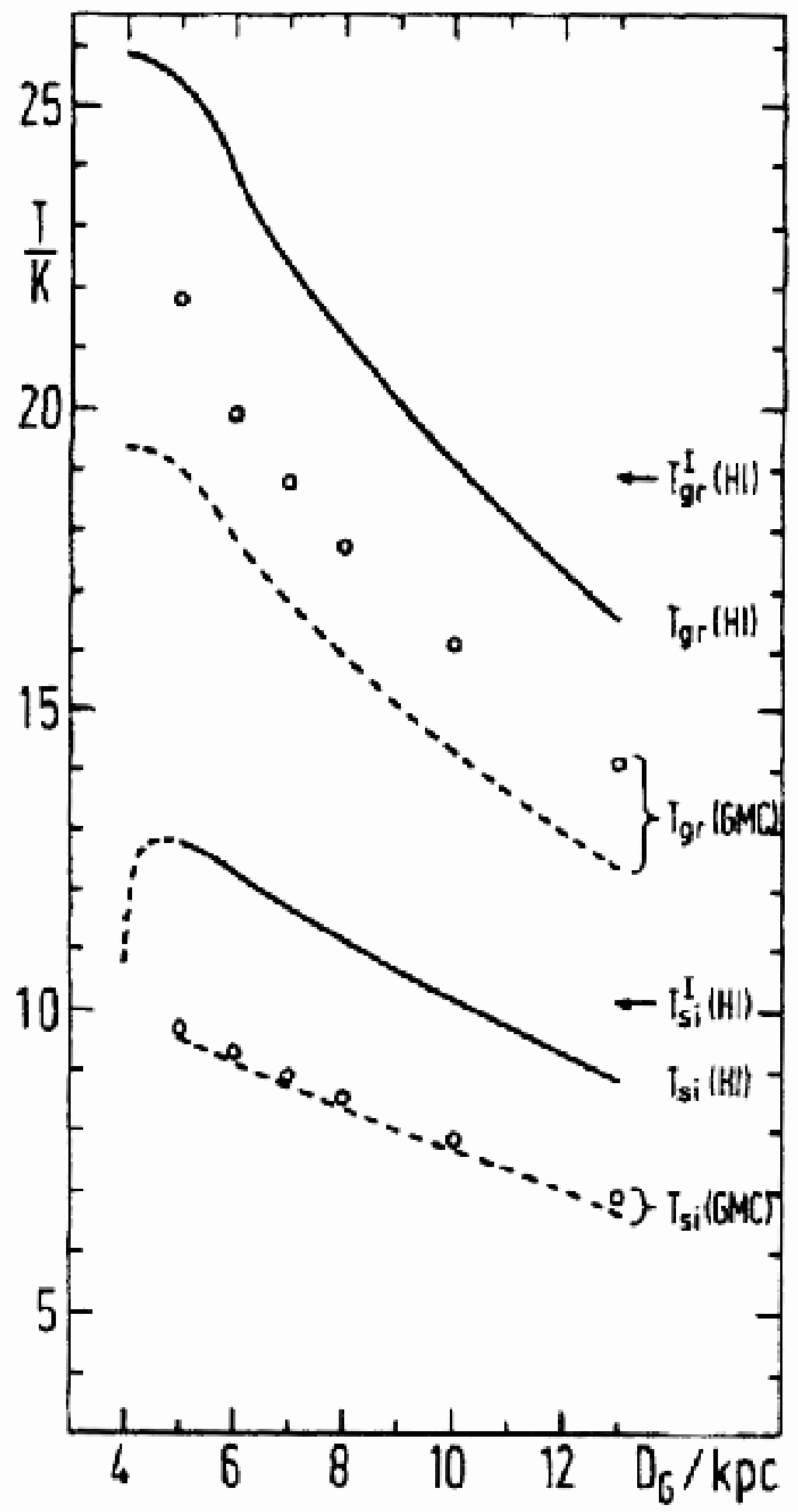

Figure 1: Temperature of dust grains in equilibrium with ISRF for various galactocentric distances given by Mathis et al. (1983); courtesy of Prof. John S. Mathis. 
At large distances, the CMB limit will be reached asymptotically. Other issues to be taken into account in a future more complete treatment of the problem of habitability of the Galaxy from the point of view of (probably postbiological) ATCs are the following:

- Cosmic ray heating, which is important even in the interiors of the densest molecular clouds (it dominates heating and ionization mechanisms there and initiates all chemical reactions in cold environments); the cosmic ray energy density at the Solar circle is about $U_{c r}=2.4 \times 10^{-12}$ erg $\mathrm{cm}^{-3}$ (Webber 1987), but falls off in a complicated manner with galactocentric distance (including Parker instability, etc.).

- Supernovae, especially of the core-collapse Type II and Type Ic, which tend to be concentrated in spiral arms and other regions of intense star-formation (for the astrobiological significance of supernovae for planetary biospheres, see e.g. Tucker and Terry 1968; Ruderman 1974; Hunt 1978; Collar 1996).

- Much rarer and more dramatic events, Galactic gamma-ray bursts (the longer ones associated with hypernovae and perhaps also shorter ones caused by neutron stars' mergers), capable of adversely influencing planetary biospheres over a large part of the Galaxy (e.g. Thorsett 1995; Scalo and Wheeler 2002; Dar and De Rújula 2002; Melott et al. 2004; Thomas et al. 2005). Possible Galactic nuclear activity falls in the same category (see below).

- Other thermodynamical issues related to computational needs, notably bit-erasure costs, as well as bandwidth and latency issues (Sandberg 2000).

It is significant that both radiative and kinetic energy inputs from supernovae and related events are adverse to the computation efficiency of ATCs. All these effects are falling off with the galactocentric distance, and become very small for $R>15 \mathrm{kpc}$. In the inner parts of the Galaxy, the same factors which preclude habitability (mainly supernovae and gamma-ray bursts) act to preclude computation as well. In addition, the issue of the nuclear activity of the Milky Way and spiral galaxies in general, may be important for astrobiological evolution of those regions. It has been proposed by Clarke (1981) in an interesting early paper, as a mechanism of global regulation preventing life and intelligence from arising in the entire Galaxy; see also Clube (1978) and LaViolette (1987). Although it seems now that the original idea is implausible in light of the specific conditions in Milky Way's nucleus, we should still be cautious, since the recent research unveiled tremendous nuclear outbursts in distant objects (e.g. McNamara et al. 2005). 


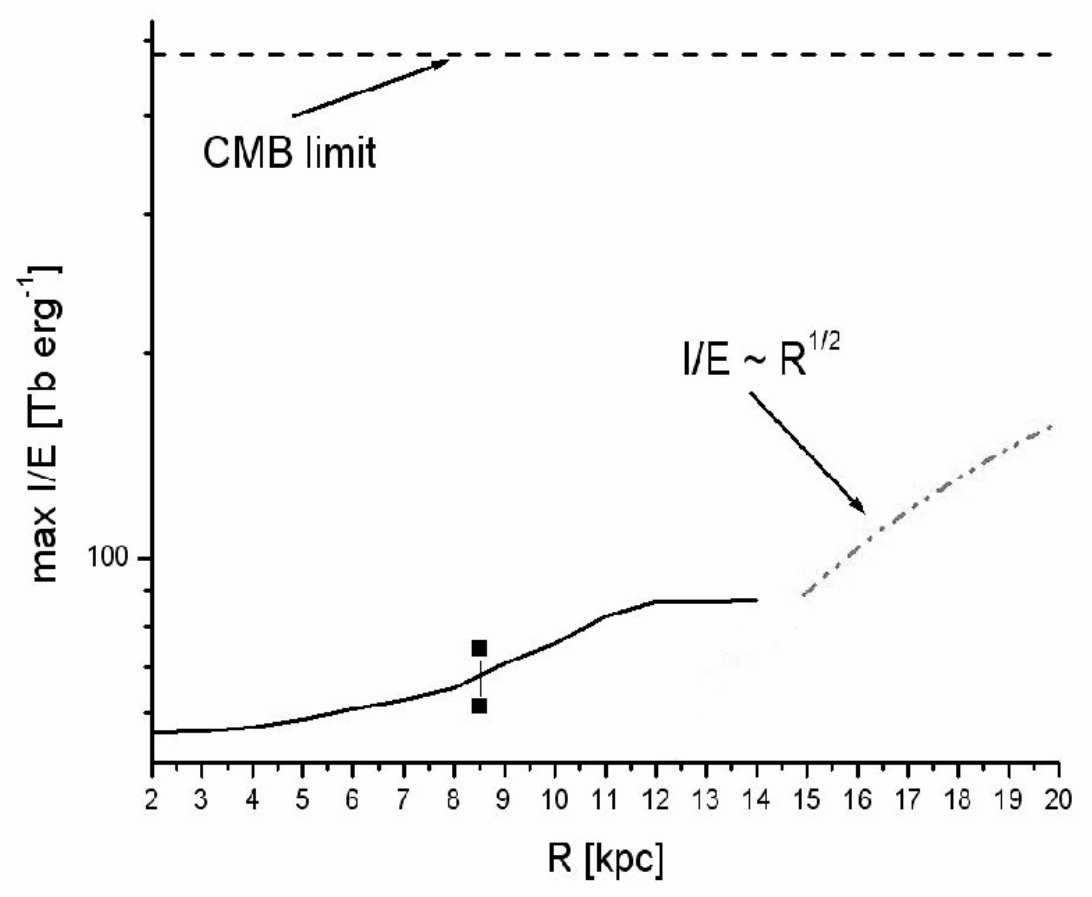

Figure 2: Maximal quantity of information per unit expended energy processed by a computer in equilibrium with the radiation field at various galactocentric distances in the Milky Way (with the location of the Solar System indicated by a vertical bar). The limit due to the cosmic microwave background is also given. We see that the efficiency limit rises considerably for $R>10 \mathrm{kpc}$. At large galactocentric distances, a simple scaling argument indicates that the efficiency will tend to rise $\propto \sqrt{R}$ until it reaches the CMB limit in the "true" intergalactic space; however, those external regions are almost devoid of baryonic matter, and even the density of cold dark matter (CDM) particles becomes exceedingly small. 
There is another large-scale gradient in the Milky Way recently firmly established by observations: the metallicity gradient. Average metal abundances of stars and ISM are rather well described by the radial gradient of $\nabla Z \simeq 0.07 \mathrm{dex} / \mathrm{kpc}$ (Hou, Prantzos, and Boissier 2000; Tadross 2003). It could seem at first that this is adverse for ATCs in the outer regions of the Galaxy, thus counteracting the trend of increasing computing efficiency described above. But, the Intelligence Principle suggests something different, when we take into account that the chemical enrichment causing the gradient is entirely the product of stellar nucleosynthesis; primordial composition was uniformly metal-free. Stellar nucleosynthesis is appallingly inefficient process by ATC standards; it converts $\sim 1 \%$ of the rest mass to energy and most of that created energy left the Galaxy a long time before the emergence of first ATCs. Thus, baryonic matter in the primordial composition would, in principle, be desirable for advanced optimization of computation. ATCs would subsequently be able to create heavier nuclei by controlled nuclear fusion and minimize the energy leak per unit of created entropy.

\section{Migration hypothesis}

Taking all this into account, we suggest the "migration hypothesis": ATCs will tend to move their computing facilities toward the colder regions of the Milky Way in order to make their information processing as efficient as possible. In general case, this would mean the outskirts of the Galaxy, but the interiors of the giant molecular clouds could also serve as local foci for the advanced information processing. ${ }^{5}$ If the postbiological evolution is predominant, as suggested by Dick (2003) and other recent authors, this would mean that the entire ATC will tend to migrate outward from its original location in the GHZ toward a convenient location in the Galactic "technological zone" (GTZ) with temperature low enough to increase computing efficiency. Although such a migration will seem expensive at first glance, it is not necessarily so: postbiological civilizations are likely to be small, compact, stable over astrophysical timescales and would be able to travel as redundant information storages at small speeds with negligible energy expenditures. Almost all energy will be needed for acceleration and deceleration. Starting at a particular galactocentric distance, it is not difficult to calculate that any specific transportation cost will be covered by increased computation efficiency on timescales short compared to the astrophysical timescales (stellar Main Sequence lifetimes; cf. Zuckerman 1985) or even the timescale of the travel itself! Interestingly enough, bold suggestions for the possible technologies

\footnotetext{
${ }^{5}$ Insofar as the other risks, from the point of view of ATCs and the Intelligence Principle, mentioned above can be avoided: notably star-formation bursts and associated Type II supernovae.
} 
of such interstellar migrations already exist in the literature, notably in the form of "stellar engines" (e.g. Badescu and Cathcart 2000) or gravitational assists (Prado 1996; Vulpetti 1999).

What limits the outward migration of ATCs? This is largely a contextdependent issue, but the most plausible limit is set simply by available supply of matter. Depending on whether ATCs get to use non-baryonic dark matter, whose density roughly varies in accordance with the isothermal profile ( $\rho \propto$ $R^{-2}$ ), or only baryonic matter, which falls off exponentially (6), the maximal distance a cost-mindful ATC may be located at will greatly vary. But in each case, it is a well-defined value, which limits the Galactic technological zone from the outside. The concept of GTZ should not be understood as strict and immutable: it just indicates higher relative density of "technologized" matter than elsewhere. ATCs can arise and function elsewhere (in the same sense as life can arise outside of the classical circumstellar habitable zone; e.g. in Europa-like subglacial oceans), but the probability of finding them is not uniform; on the present hypothesis, the maximum probability will be located in the ring on the periphery of the Milky Way.

It is important to understand that while we do not doubt that ATCs will eventually have astro-engineering means to prevent any individual catastrophic occurrences like supernovae or GRBs, ${ }^{6}$ we doubt that it can ever become worthwhile to manage and police the Galaxy in this manner. Energy/information and time cost are likely to remain too high in all epochs. On the contrary, it seems probable that any rational cost-benefit analysis would favor migration to the Galactic rim rather than the costly and risky "policing" strategy.

Migration in physical space will be analogous to the prior migration of the bulk of civilization's interests and pursuits from physical to digital space. This presents an additional factor helping explain Fermi's paradox: advanced civilizations based on an optimized computronium (i.e. whatever substrate upon which computation can take place) infrastructure have little need for conversations with human-level individuals or even civilizations whose thought capacities are trillions of times less than their own (cf. Ćirković and Radujkov 2001). In contrast, they may have an interest in leaving our civilization and other "late comers" to their own unique development path so as to increase the potential diversity and information content of the Galaxy. As elaborated by Bradbury (2001), this is due to the large phase space of what can be constructed using molecular nanotechnology and the difficulties in proving that the computational architectures previously adopted to support advanced civilizations are, in fact, "optimal". ATCs may need less developed civilizations for the "dumb luck" they may have in developing an unexplored quadrant of the phase space of what may be designed and assem-

\footnotetext{
${ }^{6}$ This could be achieved through technologies already envisaged, like stellar lifting and long-term orbit modifications.
} 
bled in support of the evolution of intelligence. An additional consequence of the outward migration would be a dramatic increase in the average distance between ATCs; this circumstance will be important for assessing practical SETI prospects below.

Compared with the usual SETI assumptions, the present hypothesis favors a small, compact, highly efficient ATCs, in both biological and postbiological cases. In contrast to the usually assumed model of expanding "colonial empire" from human history (which confronts us with the gravest form of Fermi's paradox), the present picture would rather use a model of a "city-state" - if anything from the human history is to be even remotely analogous to the generic pathway of ATCs, which is itself a doubtful proposition. It is too often forgotten (both among SETI proponents, as well as the contact pessimists) that colonial expansion has been an exception, rather than the rule in human history so far; our Western-centric attitude should not blind us into accepting a wrong model for civilizational behavior. Countless city-states, be they in ancient Greece, pre-Aryan India, Babylonia, medieval Italy, Germany or Russia, pre-Incan Andes or Mayan Mexico, have all together much longer and stronger traditions than imperial powers, of which there are no more than two dozen examples altogether, from Assyria to the USA. Even in the cases where cities and other smaller organizational units have been peacefully or otherwise incorporated into a larger whole, this was often regarded as optimization of resources and management, and clear limits to growth have been set in advance; examples in this respect range from Achaean League, to Hansa, to Swiss Confederation, to China after Ch'in unification. It is exactly this understanding of limits (or resources and communication) which made the longevity of civilizations like the Chinese, or organizations like the Roman Catholic Church so prominent in the human history so far. Vice versa, it was disregard for these limits which contributed to downfalls of all historical empires.

As noticed by Gould (1989), the normative concept of "progress through conquest and displacement" is intimately linked with the Victorian "chain of being" fallacy. According to this view, all lifeforms have their exact position in the chain ranging from the most primitive Archaea to the gentlemen with white hats doing a noble job of conquering savages all around the world. This view has been abandoned in practically all fields - except, ironically enough, SETI studies. In general, SETI is mostly in the same shape and with the same set of philosophical, methodological and technological guidelines as it was in the time of its pioneers (Drake, Sagan, Shklovskii, Bracewell, Oliver, Morrison) in 1960s and 1970s. ${ }^{7}$ In contrast, our views of astrophysics, biology, and especially, computer science - arguably the three key scientific disciplines for SETI - changed revolutionarily, to put it mildly, since that epoch. The

\footnotetext{
${ }^{7}$ For a prototype "Galactic Club" optimistic - or naive - view of that epoch, exactly 30 years old, see Bracewell (1975). Early SETI literature abounds in such enthusiasm.
} 
present study is an attempt to break this mold and point serious modern alternatives to the old-fashioned SETI philosophy.

The present approach is similar to the one favored by Dyson (2003), who suggests searching for life at distant objects of the Solar System (and other planetary systems). Although Dyson only considers primitive life, this can be easily generalized to life of higher level of complexity and even intelligence. ${ }^{8}$ In his other writings, Dyson has suggested supercivilizations of various sorts whose activities can be detected even if they are not actively engaged in an effort to contact and communicate with other societies; for an early review of such ideas, see Lemarchand (1995). In our view, the migration hypothesis can solve Fermi's paradox, since the truly advanced societies, i.e. those who survive the bottleneck presented by the threat of self-destruction through warfare or accident will tend to be located at the outskirts of the Milky Way, outside of the main thrust of SETI projects so far. The very same traits making ATCs capable of migrating and utilizing resources with high efficiency (compactness, high integration, etc.) will tend to make them systematically hard to detect from afar. This is in diametrical opposition to views of many early SETI researchers - recently brought to a sort of the logical extreme by Weinberger and Hartl (2002) — that ATCs will indulge in extravagant spending in order to achieve interstellar communication, even if only nominal. The same applies mutatis mutandis to the large-scale interstellar travel to diverse targets; the nature of the postbiological megatrajectory is not likely to include any gain from the scattering of pieces of an ATC all over the Galaxy. ${ }^{9}$ One of the SETI pioneers, Benjamin Zuckerman proposed in 1985 that stellar evolution is an important motivation for civilizations to undertake interstellar migrations (Zuckerman 1985). Although arguments presented in that study seem outdated in many respects, it is significant that the mass migration idea has been presented even in the context of classical SETI studies, biological evolution and pre-digital perspective. It seems implausible that any but the most extreme conservative societies would opt to wait to be forced to migration by slow and easily predictable process like their domicile star leaving the Main Sequence.

\footnotetext{
${ }^{8}$ Parenthetically, the same line of reasoning suggests that the search for extraterrestrial artifacts (SETA) should concentrate to the outskirts of the Solar System, notably the Kuiper belt objects and even Oort cloud comets. Low-profile digital approach would warrant maximization of the information processing for the hypothetical ATC probes also.

${ }^{9}$ This is related to the possibility of postbiological ATCs being what Bradbury (2001) dubs "distributed replicated intelligence[s]". Although elaboration of this admittedly speculative concept is far beyond the scope of the present study, it is enough to mention the intuitively clear picture in which, once a threshold of complexity is reached, it is very hard to separate an intelligent part from the whole of the ATC to any distance making the latency problems due to the finite speed of light important. This may pose problems for anything but the simplest, low-profile, interstellar drones or passive inscribed matter packages.
} 
Not surprisingly, some of the ideas presented here have been forefathered in a loose form within SF discourse. Karl Schroeder in "Permanence" not only formulated an unrelated answer to Fermi's question, but, more pertinently, envisaged the entire Galaxy-wide ecosystem based on brown dwarfs (and halo population in general) and low-temperature environment (Schroeder 2002; see also Ćirković 2005). The idea of a new megatrajectory comprising "mainstream" evolution of ATCs and containing the theoretical explanation of Fermi's paradox has been beautifully discussed by Stanislaw Lem (especially 1987, but see also Lem 1984). Most strikingly, the idea of ATCs inhabiting the outer fringes of the Milky Way has been suggestedthough without the thermodynamical rationale - by Vernon Vinge in "A Fire upon the Deep" (Vinge 1991). Vinge vividly envisages "Zone boundaries" separating dead and low-tech environments from the true ATCs inhabiting regions at the boundary of the disk and high above the Galactic plane. This is roughly analogous to the low-temperature regions we outlined as the most probable Galactic technological zone.

\section{Discussion: failure of the conventional SETI perspective}

In building of the migrational solution to Fermi's puzzle, we have relied on the following set of assumptions:

1. The Copernican principle continues to hold in astrobiology, i.e. there is nothing special about the Earth and the Solar System when considerations of life, intelligent observers or ATCs are made.

2. Laws of physics (as applied to the classical computation theory and astrophysics) are universally valid.

3. Naturalistic explanations for the origin of life, intelligence and ATCs are valid.

4. The Milky Way exhibits well-established gradients of both baryonic matter density and equilibrium radiation field temperature.

5. Habitable planets occur naturally only within GHZ (which evolves in a manner roughly understood), but ATCs are not in any way limited to this region.

6. We assume local influences both of and on ATCs. Thus, we disregard speculative ideas about wormholes, "basement universes", etc. Interstellar travel is feasible, but is bound to be slow and expensive (for anything larger than nanomachines) at all epochs. 
7. Astro-engineering on the scales significantly larger than the scale of a typical planetary system (e.g., on the parsec scale and above) will remain difficult and expensive at all epochs and for all ATCs.

8. ATCs will tend to maximize efficiency of information-processing, no matter how heterogeneous their biological, cultural, etc. structures and evolutionary pathways are.

These assumptions are, of course, of varying validity and importance. Items 1, 2, and 3 are essential methodological guidelines of the entire scientific endeavor; although 1 has recently become controversial within "rare Earth" theorists' circle, there is still no compelling reasons for relinquishing it. Assumption 4 is an empirical fact, and 5 is quite close to it. Assumptions 6 and 7 are conservative extrapolations of our limited scientific and technological perspective, but in our view should be retained until the contrary positions can be verified. In particular, absence of the Galaxy-size astro-engineering effects in external galaxies (Annis 1999b) strongly supports assumption 7.

Most controversial, of course, is the culturological (or meta-ethical) assumption 8. One way to justify it is to observe the alternative long-term strategies in a given cosmological setting. Ultimately, ATCs will face the limits of cosmology and fundamental physics (Tipler 1986; Adams and Laughlin 1997; Ćirković 2004c); their vastly improved predicting capacities will enable them to obtain high-resolution models of such situations far in advance. Two limits seem reasonable: evolving into either pure pleasure seeking and hedonism in the most general sense (a "Roman empire" analogue) or onto a pathway toward the greatest accomplishments possible along their individual development vector (a "Greek Olympics" analogue). In either situation they will seek the greatest computational capacity and efficiency possible to support these activities.

We wish to re-emphasize the absence of exotic physics or inconceivably advanced technology in our analysis. Its central piece, Brillouin inequality is valid for classical computation. If much discussed (in theory) quantum computation becomes practical possibility, it might not be bound by it (although an analogous constraint, Margolus-Levitin bound might step in its place; cf. Dugić and Ćirković 2002). On the more exotic/SF side of the story, one might imagine creating wormholes to non-local sources of usable energy, or even entire "basement universes" envisaged by Linde (1990, 1992; see also Garriga et al. 2000).

The migration hypothesis smoothly joins with the global catastrophic solutions, such as those proposed by Clarke (1981) and Annis (1999a; see also Norris 2000). In those scenarios, there is a global regulation mechanism for preventing the formation of complex life forms and technological societies early in the history of the Galaxy. Such a global mechanism could have the physical form of $\gamma$-ray bursts, if it can be shown that they exhibit sufficient 
lethality to cause mass biological extinctions over a large part of the volume of the Galactic habitable zone (Scalo and Wheeler 2002; see also Thorsett 1995; Melott et al. 2004). However, since the regulation mechanism exhibits secular evolution, with the rate of catastrophic events decreasing with time, at some point the astrobiological evolution of the Galaxy will experience a change of regime. When the rate of catastrophic events is high, there is a sort of quasi-equilibrium state between the natural tendency of life to emerge, spread, diversify, and complexify, and the rate of destruction and extinctions. When the rate becomes lower than some threshold value, intelligent and space-faring species can arise in the interval between any two extinctions and make themselves immune (presumably through technological means) to further extinction/causing events. ${ }^{10}$ The migration hypothesis complements such catastrophic solutions to Fermi's puzzle, since it adds another layer to the "Great Filter" (Hanson 1998) explaining the absence of ATCs or their manifestations. Annis' and related hypotheses suggest that ATCs are both rarer and younger than we would naively expect based on uncritical gradualism; the migration hypothesis presented here indicates that even those which exist at present would be hard to detect due to their peripheral distribution, as well as other difficulties related to their postbiological evolution. In other words, not-yet-ATCs are decimated by catastrophes, while ATCs - who are immune to such contingencies - have predominantly migrated to the Galactic rim long time ago.

An objection that the proposed solution violates Occam's razor must be considered. William of Occam a 14th century English Franciscan, strongly espoused nominalism against the Platonic concept of ideal types as entities in a realm higher than material existence (a viewpoint conventionally known as realism). Occam devised his famous motto, non sunt multiplicanda entia praeter necessitatem (entities are not to be multiplied beyond necessity), as a weapon in this philosophical battle - an argument against the existence of an ideal Platonic realm (for nominalists regard names of categories only as mental abstractions from material objects, and not as descriptions of higher realities, requiring an additional set of unobserved ideal entities, or essences). Occam's razor, in its legitimate application, therefore operates as a logical principle about the complexity of an argument, not as an empirical claim that nature must be maximally simple. It is exactly this often underappreciated point which makes the present solution to Fermi's paradox actually simpler than most of the alternatives. Consider, for instance, the hypothesis that a hundred prospective (independently arising) ATCs randomly distributed over the Milky Way disk destroyed themselves through internal warfare before leaving their home planets. It is - apart from the appeal to a mystical and universal fatum - an excessively complex hypothesis, relying on expla-

\footnotetext{
${ }^{10}$ In fact, an alternative definition of ATCs may consist of the requirement that an intelligent community is immune, as a community, to all kinds of natural catastrophes.
} 
nation of the observed "Great Silence" through a hundred groups of both logically and spatio-temporally disjoint causes. Contrariwise, the migration hypothesis proposed here suggests that a fraction of these civilizations will, upon surviving the filter of natural and artificial catastrophes, essentially drop out of sight through optimization of computing resources (implying preferred peripheral distribution in the Galaxy, not wasting energy on inefficient communication, etc.). This can be, in turn, reduced to a small number of causes, essentially those presented as the assumptions 1-8 above.

Once adopted as a viable solution to Fermi's paradox, the migration hypothesis presented here has both theoretical and practical consequences. First of all, inconvenient location of most of ATCs as observed from the Solar System, coupled with realization that distinguishing signal from noise is much harder than usually thought (Lachmann et al. 2004) and may even be completely substituted by inscribed-matter messages (Rose and Wright 2004), are sufficient to explain the lack of results in SETI projects so far. Some of the SETI pioneers have been very well aware of this and warned about it (notably Sagan 1975); these cautious voices have been consistently downplayed by the SETI community. We conclude that the conventional radio SETI assuming beamed broadcasts from targets within Solar vicinity (e.g. Turnbull and Tarter 2003) is ill-founded and has little chance of success on the present hypothesis. It is a clear and testable prediction of the present hypothesis that the ongoing SETI experiments using this conservative approach will yield only negative results.

The picture sketched in the present study undermines the basic tenets of the prevailing SETI philosophy. Outward migration of advanced technological species should be taken into account in any serious SETI project. Given the likely distances of an ATCs that began migration tens of millions to billions of years ago (Lineweaver 2001), they are not likely to know of our development. While their observational capabilities probably allow them to observe the Solar System, they are looking at it before civilization developed. It is doubtful, to say the least, that they would waste resources sending messages to planetary systems possessing life, but quite uncertain (in light of the biological contingency) to develop a technological civilization. Dolphins and whales are quite intelligent and possibly even human-level conscious (e.g. Browne 2004), but they do not have the ability to detect signals from ATCs, and it is uncertain that they will ever evolve such a capacity. By a mirrorimage of such position, unless one has concrete evidence of an ATC at a given locale, it would be wasteful to direct SETI resources towards them. Ironically enough, this can offer a rationale to some of the SETI sceptics, but based on the entirely different overall astrobiological picture and with completely different practical consequences.

While fully recognizing that patience is a necessary element in any search, we still wish to argue that the conventional SETI (Tarter 2001; Duric and 
Field 2003, and references therein), as exemplified by the historical OZMA Project, as well its later counterparts (META, ARGUS, Phoenix, SERENDIP/ Southern SERENDIP, etc.), notably those conveyed by NASA and the SETI Institute, is fundamentally flawed. This is emphatically not due to the real lack of targets, us being alone in the Galaxy, as contact-pessimists in the mold of Tipler or Mayr have argued. Quite the contrary, it is due to physical reasons underlying flaws in the conventional SETI wisdom: in a sense the problem has nothing to do with the universe itself, and everything to do with our ignorance and prejudices. In this special sense, the flaws in the currently prevailing views on SETI are less excusable. ${ }^{11}$

Instead, much stronger emphasis on the ATC manifestations and traces is the only serious recourse of practical SETI. Even if they are not actively communicating with us, we could in principle detect them and their astroengineering activities. Their detection signatures may be much older than their communication signatures. Unless ATCs have taken great lengths to hide or disguise their IR detection signatures, the terrestrial observers should still be able to observe them at those wavelengths and those should be distinguishable from normal stellar spectra. The same applies to other un-natural effects, like the antimatter-burning signatures (Harris 1986, 2002), or recognizable transits of artificial objects (Arnold 2005). Search for mega-projects such as Dyson shells, Jupiter Brains or stellar engines are most likely to be successful in the entire spectrum of SETI activities (Slysh 1985; Jugaku, Noguchi, and Nishimura 1995; Jugaku and Nishimura 2003). Search for such astro-engineering traces of ATCs should be primarily conducted in the infrared part of the electromagnetic spectrum (Dyson 1960; Tilgner and Heinrichsen 1998; Timofeev, Kardashev, and Promyslov 2000). Ironically enough, surveys in the infrared have been proposed by one of the pioneers of radioastronomy, Nobel-prize winner Charles H. Townes, although on somewhat different grounds (Townes 1983). Bold and unconventional studies, such as Harris', Arnold's, Slysh's, or the survey of Jugaku et al. and program proposed by Tilgner and Heinrichsen (1998), represent still a minuscule fraction of the overall SETI research. We dare suggest that there is no real scientific reason for such situation: instead, it has emerged due to excessive conservativeness, inertia of thought, overawe of the "founding fathers", or some combination of the three. The unconventional approach with emphasis on search for ATCs' manifestations would loose nothing of the advantages of

\footnotetext{
${ }^{11}$ It is not just the present hypothesis which leads to such a conclusion. Different views on the evolution of ATCs, not based on the Intelligence Principle and the digital perspective, lead to the same general idea. For example, this applies to the ingenious idea that ATCs will transfer their cognition into their environment (Karl Schroeder, private communication), following recent studies on the distributed natural cognition (e.g. Hutchins 1996). In these, as in other suggested lines of "mainstream" ATC development, the approaches currently favored by SETI projects will be fundamentally misguided, i.e. ATCs remain undetectable by such approaches.
} 
conventional SETI before detection (e.g. Tough 1998), but the gains could be enormous. In accordance with the motto of Heraclitus, we should "expect the unexpected" if we desire genuine SETI results; otherwise, we "won't find them" (i.e. traces of ATCs).

The hypothesis presented here is falsifiable inter alia by extragalactic SETI observations. Extragalactic SETI has not been considered very seriously so far (for notable exceptions see Wesson 1990; Annis 1999b). The reason is, perhaps, the same old comforting prejudice that we should expect specific (and, conveniently, radio) signals. Since these are not likely forthcoming over intergalactic distances (and two-way communication desired by SETI pioneers is senseless here in principle), there is no point in even thinking seriously about extragalactic SETI. From the preceding, it is clear how systematically fallacious such a view is: when we remove the cozy assumption of specific SETI signals (together with the second-order assumption of their radio nature), this view collapses. On the contrary, extragalactic SETI would enable us to probe enormously larger part of physical space as well as the morphological space of possible evolutionary histories of ATCs. (Of course, part of what we get ensemble-wise we loose time- and resolution-wise.) In fact, the definition of Kardashev's Type III civilization should prompt us to consider it more carefully, at least for a sample of nearby galaxies, visible at epochs significantly closer to us than the 1.8 Gyr difference between the median age of terrestrial planets and the age of Earth (Allègre et al. 1995; Lineweaver 2001). It could be argued (although it is beyond the scope of the present study) that the null result of extragalactic SETI observations so far (Annis 1999b) represents a strong argument against the viability of Kardashev's Type III civilizations. While it remains a possibility in the formal sense of being in agreement with the known laws of physics, it seems that the type of pan-galactic civilization as envisaged by Kardashev and other early SETI pioneers is either (i) much more difficult to build (suggesting that the sample of $\sim 10^{3}$ normal spiral galaxies close enough and observed in high enough detail is simply too small to detect even a single Type III civilization), or (ii) simply not worth striving to. In contrast, the concept of spatially smaller, compact, efficient ATCs motivated by a convergent set of economic, ecological and/or ethical premises, inhabiting fringes of the luminous matter distribution presents to us more plausible alternative to the conventional Type III picture. This will remain valid even if (for some entirely different reason) the present hypothesis could not account for Fermi's paradox in the Milky Way. The true test here would be to detect signs of astro-engineering efforts at the outskirts of nearby spiral galaxies (i.e. those which are seen at about the same epoch as we are living in), and in their immediate intergalactic vicinity. Observations of the edges of spiral galaxies are notoriously difficult (e.g. Bland-Hawthorn, Freeman, and Quinn 1997), but they are rapidly improving in both quality and quantity. It is quite con- 
ceivable that they will give us the first hint about the generic fate of advanced intelligent communities.

Acknowledgements. Invaluable technical help has been received from Dušan Indjić, Branislav K. Nikolić, Saša Nedeljković, Vesna Milošević-Zdjelar, Maja Bulatović, Samir Salim, Nick Bostrom, Olga Latinović, Aleksandar Zorkić, Milan Bogosavljević, Aleksandar B. Nedeljković, and Vjera Miović. Pleasant discussions with Zoran Knežević, Irena Diklić, Ivana Dragićević, Zoran Živković, Anders Sandberg, Fred C. Adams, George Dvorsky, Petar V. Grujić, Karl Schroeder, Robin Hanson, Mašan Bogdanovski, James Hughes, Tanja Berić-Bjedov, Maja Jerinić, Mark A. Walker, Richard B. Cathcart and Viorel Badescu have immensely contributed to development of the ideas presented here. One of the authors (M. M. Ć.) has been partially supported by the Ministry of Science and Environmental Protection of Serbia through the project no. 1468, "Structure, Kinematics and Dynamics of the Milky Way"; he also uses the opportunity to thank the KoBSON Consortium of Serbian libraries, which has enabled overcoming of the gap in obtaining the scientific literature during the tragic 1990s in the Balkans.

\section{References}

Adams, F. C. and Laughlin, G. 1997, " A dying universe: the long-term fate and evolution of astrophysical objects," Rev. Mod. Phys. 69, 337372.

Allègre, C. J., Manhès, G., and Göpel, C. 1995, "The age of the Earth," Geochim. Cosmochim. Acta 59, 1445-1456.

Andrews, D. G. 2003, "Interstellar Transportation using Today's Physics," AIAA Paper 2003-4691, report to 39th Joint Propulsion Conference \& Exhibit.

Annis, J. 1999a, "An Astrophysical Explanation for the Great Silence," J. Brit. Interplan. Soc. 52, 19-22 (preprint astro-ph/9901322).

Annis, J. 1999b, "Placing a limit on star-fed Kardashev type III civilisations," J. Brit. Interplan. Soc. 52, 33-36.

Arnold, L. F. A. 2005, "Transit Lightcurve Signatures of Artificial Objects," Astrophys. J., in press (preprint astro-ph/0503580).

Asghari, N. et al. 2004, "Stability of terrestrial planets in the habitable zone of Gl 777 A, HD 72659, Gl 614, 47 UMa and HD 4208," Astron. Astrophys. 426, 353-365.

Bada, J. L. 2004, "How life began on Earth: a status report," Earth Planet. Sci. Lett. 226, 1-15. 
Badescu, V. 1995, "On the radius of Dyson's sphere," Acta Astronautica 36 135-138.

Badescu, V. and Cathcart, R. B. 2000, "Stellar engines for Kardashev's type II civilizations," J. Brit. Interplane. Soc. 53, 297-306.

Beaugé, C., Callegari, N., Ferraz-Mello, S., and Michtchenko, T. A. 2005, "Resonance and stability of extra-solar planetary systems," in $D y$ namics of Populations of Planetary Systems, Proceedings of the IAU Colloquium No. 197, ed. by Z. Kneevic and A. Milani (Cambridge University Press, Cambridge), 3-18.

Binney, J. and Merrifield, M. 1998, Galactic Astronomy (Princeton University Press, Princeton).

Bland-Hawthorn, J., Freeman, K. C. and Quinn, P. J. 1997 "Where Do the Disks of Spiral Galaxies End?" Astrophys. J. 490, 143-155.

Bostrom. N. 2000, "When Machines Outsmart Humans," Futures 35, 759764.

Bracewell, R. N. 1975, The Galactic Club: Intelligent Life in Outer Space (W. H. Freeman, San Francisco).

Bradbury, R. J. 1997, "Jupiter \& Matrioshka Brains: History \& References," preprint at http://www . aeiveos. com/ bradbury/JupiterBrains/.

Bradbury, R. J. 2001, "Matrioshka brains," preprint at http://www.aeiveos.com/ $\sim$ bradbury/MatrioshkaBrains/MatrioshkaBrains.html

Brillouin, L. 1962, Science and Information Theory (Academic Press, New York).

Brin, G. D. 1983, "The great silence - the controversy concerning extraterrestrial intelligent life," Q. Jl. R. astr. Soc. 24, 283-309.

Browne, D. 2004, "Do dolphins know their own minds?" Biology and Philosophy 19, 633-653.

Carter, B. 1983, "The anthropic principle and its implications for biological evolution," Philos. Trans. R. Soc. London A 310, 347-363.

Cavicchioli, R. 2002, "Extremophiles and the Search for Extraterrestrial Life," Astrobiology 2, 281-292.

Chernavskii, D. S. 2000, "The origin of life and thinking from the viewpoint of modern physics," Physics-Uspekhi 43, 151-176.

Clarke, J. N. 1981, "Extraterrestrial Intelligence and Galactic Nuclear Activity," Icarus 46, 94-96.

Clube, S. V. M. 1978, "Does our galaxy have a violent history?" Vistas in Astronomy 22, 77-118.

Cohen, J. and Stewart, I. 2002, What Does a Martian Look Like? (John Wiley \& Sons, Hoboken, New Jersey).

Collar, J. I. 1996, "Biological Effects of Stellar Collapse Neutrinos," Phys. Rev. Lett. 76, 999-1002. 
Cox, P., Krügel, E., and Mezger, P. G. 1986, "Principal heating sources of dust in the galactic disk," Astron. Astrophys. 155, 380-396.

Criswell, D. 1985, "Solar system industrialization: Implications for interstellar migration," in Interstellar Migration and the Human Experience, ed. by B. Finney and E. Jones (University of California Press, Berkeley), 50-87.

Ćirković, M. M. 2004a, "Earths - Rare in Time, Not Space?" J. Brit. Interplan. Soc. 57, 53-59.

Ćirković, M. M. 2004b, "On the Temporal Aspect of the Drake Equation and SETI," Astrobiology 4, 225-231.

Ćirković, M. M. 2004c, "Forecast for the Next Eon: Applied Cosmology and the Long-Term Fate of Intelligent Beings," Found. Phys. 34, 239-261.

Ćirković, M. M. 2005, "'Permanence' - An Adaptationist Solution to Fermi's Paradox?" J. Brit. Interplan. Soc. 58, 62-70.

Ćirković, M. M. and Radujkov, M. 2001, "On the Maximal Quantity of Processed Information in the Physical Eschatological Context," Serb. Astron. J. 163, 53-56.

Dar, A. and De Rújula, A. 2002 "The threat to life from Eta Carinae and gamma-ray bursts," in Astrophysics and Gamma Ray Physics in Space, ed. by A. Morselli and P. Picozza (Frascati Physics Series Vol. XXIV), pp. 513-523.

Dick, S. J. 2003, "Cultural Evolution, the Postbiological Universe and SETI," Int. J. Astrobiology 2, 65-74.

Drexler, K. E. 1992, "Molecular Manufacturing for Space Systems: An Overview," J. Brit. Interplan. Soc. 45, 401-405.

Dugić, M. and Ćirković, M. M. 2002, "Quantum Information Processing: The Case of Vanishing Interaction Energy," Phys. Lett. A 302, 291-298.

Duric, N. and Field, L. 2003, "On the Detectability of Intelligent Civilizations in the Galaxy," Serb. Astron. J. 167, 1-11.

Dyson, F. J. 1960, "Search for Artificial Stellar Sources of Infrared Radiation," Science 131, 1667-1668.

Dyson, F. J. 2003, "Looking for life in unlikely places: reasons why planets may not be the best places to look for life," Int. J. Astrobiology 2, 103-110.

Dyson, J. E. \& Williams, D. A. 1980, Physics of the interstellar medium (Manchester University Press, Manchester).

Ehrenfreund, P. et al. 2002, "Astrophysical and astrochemical insights into the origin of life," Rep. Prog. Phys. 65, 1427-1487.

Garriga, J., Mukhanov, V. F., Olum, K. D., and Vilenkin, A. 2000, "Eternal Inflation, Black Holes, and the Future of Civilizations," Int. J. Theor. Phys. 39, 1887-1900. 
Gonzalez, G., Brownlee, D., and Ward, P. 2001, "The Galactic Habitable Zone: Galactic Chemical Evolution," Icarus 152, 185-200.

Gould, S. J. 1989, Wonderful Life: The Burgess Shale and the Nature of History (W. W. Norton, New York).

Hanson, R. 1998, "The great filter - are we almost past it?" preprint available at http://hanson.gmu.edu/greatfilter.html (1998).

Harris, M. J. 1986, "On the detectability of antimatter propulsion spacecraft," Astrophys. Space Sci. 123, 297-303.

Harris, M. J. 2002, "Limits from CGRO/EGRET data on the use of antimatter as a power source by extraterrestrial civilizations," J. Brit. Interplan. Soc. 55, 383-393.

Hou, J. L., Prantzos, N., and Boissier, S. 2000, "Abundance gradients and their evolution in the Milky Way disk," Astron. Astrophys. 362, 921-936.

Hunt, G. E. 1978, "Possible climatic and biological impact of nearby supernovae," Nature 271, 430-431.

Hutchins, E. 1996, Cognition in the Wild (MIT Press, Boston).

Jugaku, J., Noguchi, K., and Nishimura, S. 1995, " A search for Dyson spheres around late-type stars in the Solar neighborhood," in Progress in the Search for Extraterrestrial Life, ed. by G. Seth Shostak (ASP Conference Series, San Francisco), 381-185.

Jugaku, J. and Nishimura, S. 2003, "A Search for Dyson Spheres Around Late-type Stars in the Solar Neighborhood," in Bioastronomy 2002: Life Among the Stars, Proceedings of IAU Symposium \# 213, ed. by R. Norris and F. Stootman (ASP Conference Series, San Francisco), 437-438.

Kardashev, N. S. 1964, "Transmission of information by extraterrestrial civilizations," Sov. Astron. 8, 217-220.

Knoll, A. H. and Bambach, R. K. 2000, "Directionality in the history of life: diffusion from the left wall or repeated scaling of the right?" in Deep Time: Paleobiology's Perspective, ed. by D. H. Erwin and S. L. Wing (The Paleontological Society, Lawrence, Kansas), 1-14.

Korycansky, D. G., Laughlin, G., and Adams, F. C. 2001, "Astronomical engineering: a strategy for modifying planetary orbits," Astrophys. Space Sci. 275, 349-366.

Kuiper, T. B. H. and Brin, G. D. 1989, "Resource Letter ETC-1: Extraterrestrial Civilization," Am. J. Phys. 57, 12-18.

Lachmann, M., Newman, M. E. J., and Moore, C. 2004, "The physical limits of communication, or Why any sufficiently advanced technology is indistinguishable from noise," Am. J. Phys. 72, 1290-1293.

Lahav, N., Nir, S., and Elitzur, A. C. 2001, "The emergence of life on Earth," Progress in Biophysics $\&$ Molecular Biology 75, 75-120. 
Landauer, R. 1961, "Irreversibility and Heat Generation in the Computing Process," IBM J. Res. Develop. 5, 183-191.

LaViolette, P. A. 1987, "Cosmic-ray volleys from the Galactic center and their recent impacts on the Earth environment," Earth, Moon and Planets 37, 241-286.

Lem, S. 1984, His Master's Voice (Harvest Books, Fort Washington).

Lem, S. 1987, Fiasco (Harcourt, New York).

Lemarchand, G. A. 1995, "Detectability of Extraterrestrial Technological Activities," SETIQuest 1, 3-13 (see electronic version at http://www . coseti.org/lemarch1.htm).

Linde, A. D. 1990, Inflation and Quantum Cosmology (Academic Press, San Diego).

Linde, A. 1992, "Stochastic approach to tunneling and baby universe formation," Nuclear Physics B 372, 421-442.

Lineweaver, C. H. 2001, "An Estimate of the Age Distribution of Terrestrial Planets in the Universe: Quantifying Metallicity as a Selection Effect," Icarus 151, 307-313.

Lineweaver, C. H. and Davis, T. M. 2002, "Does the Rapid Appearance of Life on Earth Suggest that Life Is Common in the Universe?" Astrobiology 2, 293-304.

Lineweaver, C. H., Fenner, Y., and Gibson, B. K. 2004, "The Galactic Habitable Zone and the Age Distribution of Complex Life in the Milky Way," Science 303, 59-62.

Livio, M. 1999, "How rare are extraterrestrial civilizations, and when did they emerge?" Astrophys. J. 511, 429-431.

Lloyd, S. 2000, "Ultimate physical limits to computation," Nature $4 \mathbf{4 0 6}$, 10471054.

Mathis, J. S., Mezger, P. G., and Panagia, N. 1983, "Interstellar radiation field and dust temperatures in the diffuse interstellar matter and in giant molecular clouds," Astron. Astrophys. 128 212-229.

McInnes, C. R. 2002, "Astronomical Engineering Revisited: Planetary Orbit Modification Using Solar Radiation Pressure," Astrophys. Space Sci. 282, 765-772.

McNamara, B. R., Nulsen, P. E. J., Wise, M. W., Rafferty, R. A., Carilli, C., Sarazin, C. L., and Blanton, E. L. 2005, "The heating of gas in a galaxy cluster by X-ray cavities and large-scale shock fronts," Nature 433, 45-47.

Melott, A. L. et al. 2004, "Did a gamma-ray burst initiate the late Ordovician mass extinction?" Int. J. Astrobiol. 3, 55-61.

Mezger, P. G., Mathis, J. S., and Panagia, N. 1982, "The Origin of the Diffuse Galactic Far Infrared and Sub-millimeter Emission," Astron. Astrophys. 105, 372-388. 
Minsky, M. 1973, in Communication with Extraterrestrial Intelligence (CETI), ed. by C. Sagan (MIT Press, Cambridge).

Mojzsis, S. J., Arrhenius, G., McKeegan, K. D., Harrison, T. M., Nutman, A. P., and Friend, C. R. L. 1996, "Evidence for life on Earth before 3800 million years ago," Nature 384, 55-59.

Moravec, H. P. 1988, Mind Children: The Future of Robot and Human Intelligence (Harvard University Press, Cambridge).

Noble, M., Musielak, Z. E., Cuntz, M. 2002, "Orbital Stability of Terrestrial Planets inside the Habitable Zones of Extrasolar Planetary Systems," Astrophys. J. 572, 1024-1030.

Norris, R. P. 2000, "How old is ET?" in When SETI Succeeds: The impact of high-information Contact, ed. A. Tough (Foundation for the Future, Washington DC), pp. 103-105.

Parkinson, B. 2005, "The carbon or silicon colonization of the universe?" $J$. Brit. Interplan. Soc. 58, 111-116.

Phoenix, C. and Drexler, K. 2004, "Safe exponential manufacturing," Nanotechnology 15, 869-872.

Prado, A. F. B. D. A. 1996, "Powered swingby," Journal of Guidance, Control, and Dynamics 19, 1142-1147.

Rose, C. and Wright, G. 2004, "Inscribed matter as an energy-efficient means of communication with an extraterrestrial civilization," Nature 431, 47-49.

Ruderman, M. A. 1974, "Possible consequences of nearby supernova explosions for atmospheric ozone and terrestrial life," Science 184, 10791081.

Sagan, C. 1975, "The recognition of extraterrestrial intelligence," Proc. $R$. Soc. Lond. B 189, 143-153.

Sandberg, A. 1999, "The physics of information processing superobjects: daily life among the Jupiter brains," J. Evol. Tech. 5 (http://www. jetpress.org/index.html).

Scalo, J. and Wheeler, J. C. 2002, "Astrophysical and astrobiological implications of gamma-ray burst properties," Astrophys. J. 566, 723-737.

Schaller, R. R. 1997, "Moore's law: past, present, and future," IEEE Spectrum, June 1997, 53-59.

Schroeder, K. 2002, Permanence (Tor Books, New York).

Slysh, V. I. 1985, "A search in the infrared to microwave for astroengineering activity," in The Search for Extraterrestrial Life: Recent Developments, ed. by M. D. Papagiannis (IAU, Reidel Publishing Co., Dordrecht), 315-319.

Tadross, A. L. 2003, "Metallicity distribution on the galactic disk," New Ast. 8, 737-744.

Tarter, J. 2001, "The Search for Extraterrestrial Intelligence (SETI)," Annu. Rev. Astron. Astrophys. 39, 511-548. 
Thomas, B. C., Jackman, C. H., Melott, A. L., Laird, C. M., Stolarski, R. S., Gehrels, N., Cannizzo, J. K., and Hogan, D. P. 2005, "Terrestrial ozone depletion due to a Milky Way gamma-ray burst," Astrophys. J. 622, L153-L156.

Thorsett, S. E. 1995, "Terrestrial implications of cosmological gamma-ray burst models," Astrophys. J. 444, L53-L55.

Tilgner, C. N. and Heinrichsen, I. 1998, "A program to search for Dyson spheres with the infrared space observatory," Acta Astronautica 42, 607-612.

Timofeev, M. Yu., Kardashev, N. S., and Promyslov, V. G. 2000, "A search of the IRAS database for evidence of Dyson Spheres," Acta Astronautica 46, 655-659.

Tipler, F. J. 1986, "Cosmological limits on computation," Int. J. Theor. Phys. 25, 617-661.

Tough, A. 1998, "Positive consequences of SETI before detection," Acta Astronautica 42, 745-748.

Townes, C. H. 1983, "At what wavelengths should we search for signals from extraterrestrial intelligence?" Proc. Natl. Acad. Sci. USA 80,11471151.

Tucker, W. H. and Terry, K. D. 1968, "Cosmic Rays from Nearby Supernovae: Biological Effects," Science 160, 1138-1139.

Turnbull, M. C. and Tarter, J. C. 2003, "Target Selection for SETI. I. A Catalog of Nearby Habitable Stellar Systems," Astrophys. J. 145, 181-198.

Vinge, V. 1991, A Fire upon the Deep (Millenium, London).

Vulpetti, G. 1999, "On the viability of the interstellar flight," Acta Astronautica 44, 769-792.

Ward, P. D. and Brownlee, D. 2000, Rare Earth: Why Complex Life Is Uncommon in the Universe (Springer, New York).

Ward, P. D. and Brownlee, D. 2002, The Life and Death of Planet Earth: How the New Science of Astrobiology Charts the Ultimate Fate of Our World (Henry Holt and Company, New York).

Webb, S. 2002, Where is Everybody? Fifty Solutions to the Fermi's Paradox (Copernicus, New York).

Webber, W. R. 1987, "The interstellar cosmic ray spectrum and energy density. Interplanetary cosmic ray gradients and a new estimate of the boundary of the heliosphere," Astron. Astrophys. 179, 277-284.

Weinberger, R. and Hartl, H. 2002, "A search for 'frozen optical messages' from extraterrestrial civilizations," Int. J. Astrobiology 1, 71-73.

Wesson, P. S. 1990, "Cosmology, Extraterrestrial Intelligence, and a Resolution of the Fermi-Hart Paradox," Q. Jl. R. astr. Soc. 31, 161-170.

Wilson, P. A. 1994, "Carter on Anthropic Principle Predictions," Brit. J. Phil. Sci. 45, 241-253. 
Wright, E. L. et al. 1994, "Interpretation of the COBE FIRAS CMBR Spectrum," Astrophys. J. 420, 450-456.

Zuckerman, B. 1985, "Stellar Evolution: Motivation for Mass Interstellar Migrations," Q. Jl. R. astr. Soc. 26, 56-59. 